\title{
Epidermal Cell Surface Structure and Chitin-Protein Co-assembly Determine Fiber Architecture in the Locust Cuticle
}

\author{
Sanja Sviben, Oliver Spaeker," Mathieu Bennet," Marie Albéric, Jan-Henning Dirks, Bernard Moussian, \\ Peter Fratzl, Luca Bertinetti,* and Yael Politi*
}

Cite This: ACS Appl. Mater. Interfaces 2020, 12, 25581-25590

Read Online

ACCESS

Wll Metrics \& More

国 Article Recommendations

Supporting Information

ABSTRACT: The geometrical similarity of helicoidal fiber arrangement in many biological fibrous extracellular matrices, such as bone, plant cell wall, or arthropod cuticle, to that of cholesteric liquid mesophases has led to the hypothesis that they may form passively through a mesophase precursor rather than by direct cellular control. In search of direct evidence to support or refute this hypothesis, here, we studied the process of cuticle formation in the tibia of the migratory locust, Locusta migratoria, where daily growth layers arise by the deposition of fiber arrangements alternating between unidirectional and helicoidal structures. Using focused ion beam/scanning electron microscopy (FIB/SEM) volume imaging and scanning X-ray scattering, we show that the epidermal cells determine an initial fiber orientation, from which the final architecture emerges by the self-organized co-assembly of chitin and proteins. Fiber orientation in the locust cuticle is therefore determined by both active and passive processes.

KEYWORDS: extracellular matrices, liquid crystal, chitin, protein, microvilli

$\mathrm{T}$ he orientation of fibers in extracellular matrices (ECMs), skeletal and connective tissues strongly affects their physical properties, which in turn determines morphogenesis, cell adhesion, and functionality. ${ }^{1}$ Fiber orientation therefore needs to be spatially and temporally controlled by the tissue that produces the ECM (e.g., refs 5-7). For many biological systems, it is however still unclear how the control over fiber orientation is achieved. Biological fibrous materials, such as bone, plant cell wall, and arthropod cuticle, are often described as liquid crystal (LC) analogues as they exhibit structural similarity to LC but are solid in their functional state. ${ }^{2}$ In unidirectional fiber arrangement (analogous to aligned nematic LC), sheets made of parallel fibers are stacked on top of each other with the same orientation, whereas in the helicoidal arrangement, also termed twisted plywood or Bouligand structure (analogous to cholesteric, or chiral nematic LC), the orientation of the fibers in successive sheets is continuously rotating. ${ }^{2}$ Both types of fiber orientations can be found in arthropod cuticle made of $\alpha$-chitin fibers embedded in a protein matrix. They are also present in plant cell wall made of cellulose fibers embedded in a mixed matrix of hemicellulose and lignin and in mineralized or unmineralized collagen-based tissues in vertebrates. ${ }^{2-4}$

Furthermore, many bio-macromolecules and biological crystals, among them hemicellulose, cellulose, chitin, collagen, and silk, have been demonstrated to form LC phases in vitro (reviewed in Mitov ${ }^{5}$ ). These observations have led to the hypothesis that fibrous biological materials organize by molecular self-assembly via a liquid mesophase that subsequently solidifies by dehydration and cross-linking., ${ }^{2,-8}$ Selfassembly is thought to be facilitated by matrix components such as hemicellulose in the case of plant cell wall or proteins in the arthropod cuticle. ${ }^{3}$ The latter is supported by molecular genetics studies in several insect species showing cuticle with modified fiber organization, often with lethal phenotypes, following a genetic knockdown or silencing of certain cuticular proteins. $^{\text {9-13 }}$

In contrast, evidence for cell-directed fiber organization ${ }^{14}$ and for the transfer of mechanical stresses from the cytoskeleton into the ECM, as observed in fibroblast tissue culture ${ }^{15}$ call the generality of the self-assembly hypothesis into question. In addition, although formation of chiral nematic phases of chitin nanocrystals is readily attained in vitro, the aligned nematic phase has only been achieved after application of external shearing forces to an initially chiral nematic suspension. ${ }^{16}$ Therefore, in an attempt to reconcile self-assembly and cellular control mechanisms for ECM

Received: March 10, 2020

Accepted: April 28, 2020

Published: April 28, 2020 

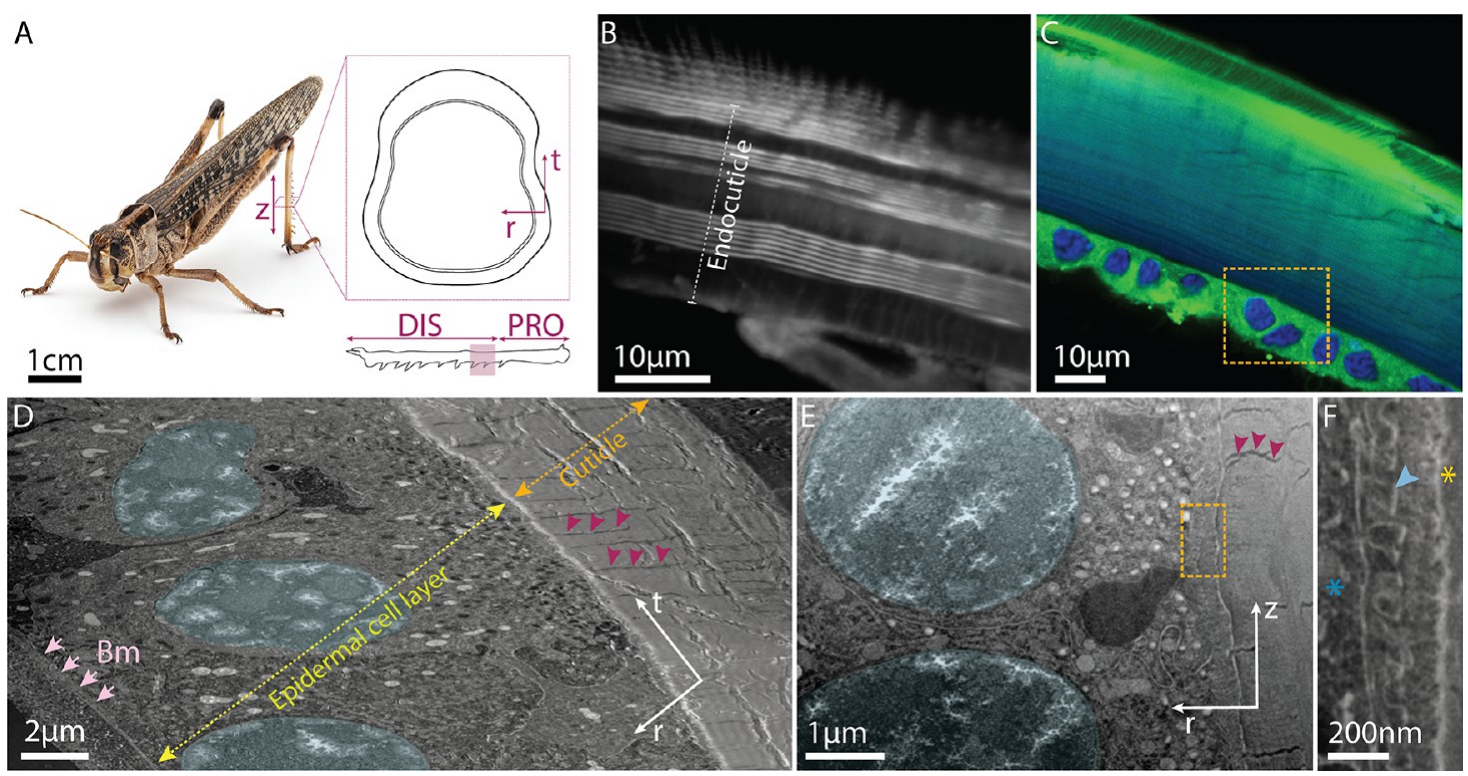

Figure 1. Cuticle deposition in L. migratoria. (A) Adult locust (AIDAsign-stock.adobe.com, reprinted with permission). Inset showing a cross section of the hind tibia and the coordinate system used throughout: $(z)$ longitudinal, $(r)$ radial, and $(t)$ transverse directions. Hind tibia of $L$. migratoria contains proximal (PRO) and distal (DIS) parts. Area marked in magenta, upper region of distal part, was sampled and analyzed in this study. (B) Confocal light scanning microscopy (CLSM) image of tibia cross section stained with Direct Yellow 96 stain showing the daily growth layers of chitin fibers in unidirectional and helicoidal fiber arrangements, leading to, respectively, alternating nonlamellate and lamellate layers. (C) CLSM tibia cross section double stained with 4',6-diamidino-2-phenylindole (DAPI) (blue) and Nile red (green) showing the epidermal cell layer adjacent to the cuticle. Orange rectangle indicates similar area of the cross section imaged using FIB/SEM in (D). (D) FIB/SEM slice showing epidermal cells between the basal membrane $(\mathrm{Bm})$ and the cuticle in fifth instar. The nuclei are false colored in light blue and the pore canals are indicated with magenta arrowheads. (E) FIB/SEM slice showing the assembly zone between the cuticle (right) and the surface of the epidermal cell layer (left) in fifth instar. Magenta arrowheads point to a pore canal; cell nuclei are false colored in light blue. The orange rectangle marks an area from which the micrograph shown in (F) is taken. (F) Magnification of the assembly zone in a region indicated in (E). The micrograph is obtained from a different depth in $t$ direction than shown in (E). The microvillar structures on the apical cell surface, which end with bright contrast at the location of the presumably chitin synthesizing plaques (light blue arrowhead), can be seen, as well as the newly deposited cuticle (yellow asterisk). Cell interior is marked with blue asterisk.

deposition, Bouligand ${ }^{17}$ suggested that cell membranes could create strong boundary conditions that influence fiber organization and self-assembly. Neville ${ }^{18}$ suggested a twomodel system with varying degrees of cellular control in the deposition of unidirectional and helicoidal architectures in insect cuticle. His suggestion was based on the observation that the unidirectional organization in insects is bilateral symmetric, while the helicoidal organization is bilateral asymmetric; the helicoidal twist is always left-handed. Here, we sought to understand how the variation in cellular control on fiber architecture is achieved. We studied the circadian clock regulated cuticle deposition in the locust, Locusta migratoria. In this species, endocuticle formation involves alternating deposition of nonlamellate layers of unidirectional fiber arrangement during the day and lamellate cuticle made of twisted plywood structure during the night (Figure 1). ${ }^{19}$ By means of two- (2D) and three-dimensional (3D) machine learning analysis of focused ion beam/scanning electron microscopy (FIB/SEM) volumes and high-resolution scanning $\mathrm{X}$-ray scattering methodologies, we show that microvilli organization at the epidermal cell surface determines an initial fiber orientation from which chitin-protein co-assembly leads to the final fiber architecture in the locust cuticle.

\section{RESULTS AND DISCUSSION}

The innermost part of the procuticle of L. migratoria (Figure $1 \mathrm{~A})$, termed endocuticle, is deposited after ecdysis by a single layer of epidermal cells that secrete the cuticle's components into a $0.5-1 \mu \mathrm{m}$ wide "assembly zone" (Figure $1 \mathrm{~B}-\mathrm{F}){ }^{20}$ Chitin is synthesized and secreted by a transmembrane enzyme, chitin synthase, located within "plaques" at the tips of microvilli emerging at the apical cell surface (Figure 1F), while cuticular proteins are proposed to be delivered to the assembly zone by vesicles. ${ }^{20}$ We imaged the deposition zone in hind tibia cuticle of animals at different developmental stages (see the Methods section) 2 or 3 days after ecdysis, collected during either the light or dark phase, in alternating $12 / 12 \mathrm{~h}$ cycle illumination conditions (hereafter termed Day or Night samples, respectively). In particular, we used FIB/SEM volume imaging to determine the spatial relationships of the microvilli with respect to each other (Figure 2), to the fibers within the assembly zone and within the cuticle (Figure 3), and to the cuticle surface (Figure 5). For segmentation and quantification, we implemented a three-dimensional machine learning algorithm based on a U-shaped, fully convolutional network (3D-FCN, see the Supporting Information for details) as proposed recently. $^{21}$

Microvilli Organization. Cryofixed samples were prepared by high-pressure freezing (HPF) cross sections of tibiae from Day and Night specimens, followed by fixation, staining, and embedding using an automatic freeze-substitution (AFS) machine. Day and Night samples show differences in the microvilli structure and organization (Figures 2 and 3). In Night, we mainly observed individual microvilli structures, with close-to-circular cross section, containing a single plaque at their tips (Figures $2 \mathrm{~A}, \mathrm{~B}$ and $3 \mathrm{~A}, \mathrm{~B}$ ). In Day, the bases of 2-4 


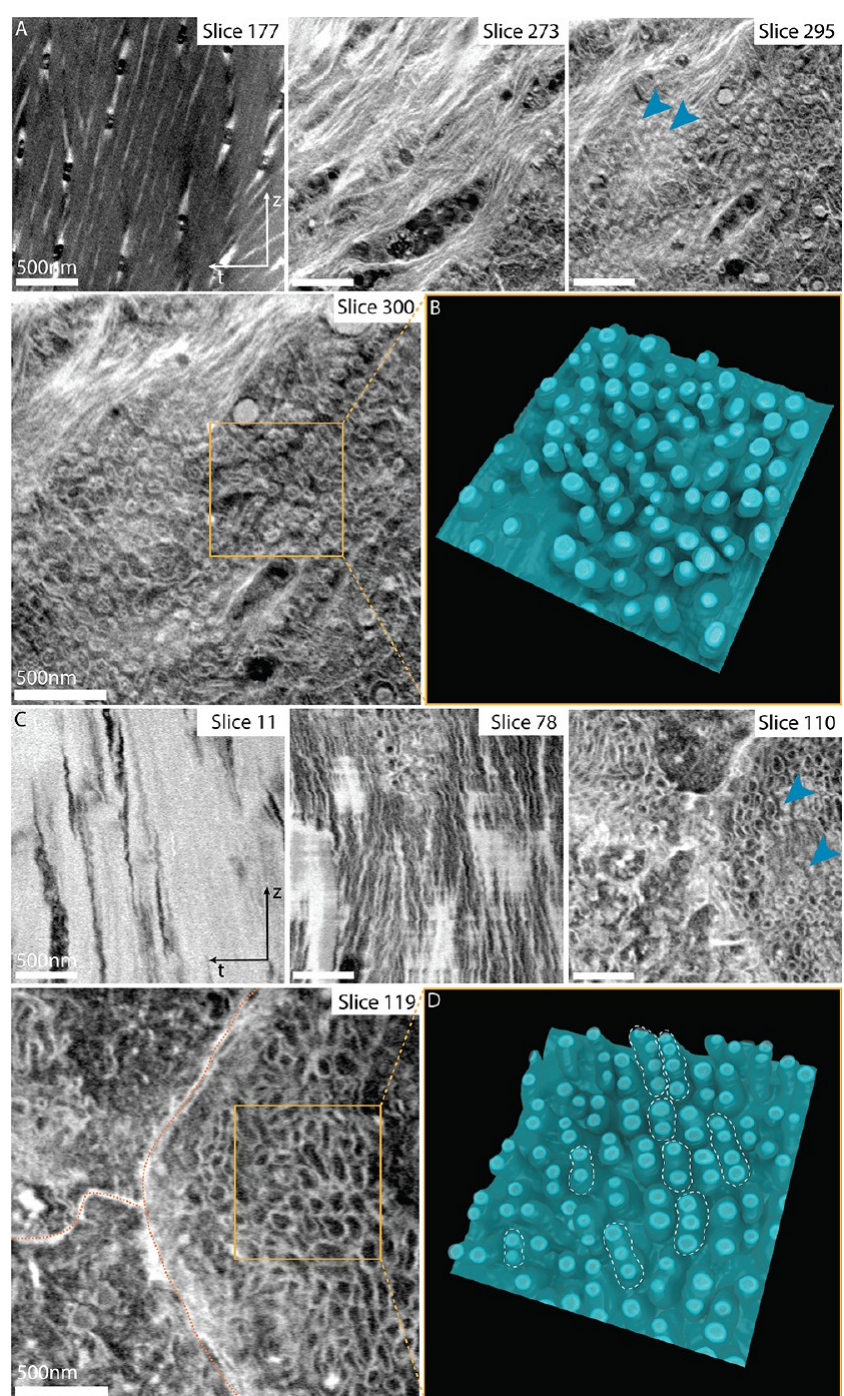

Figure 2. FIB/SEM and reconstructed 3D volume of locust cuticle and apical surface of epidermal cells. (A) FIB/SEM slices of Night and (C) Day samples of cryofixed locust tibiae obtained from adult and fifth instar specimen, respectively, 2 days after ecdysis. Reconstruction of microvilli in Night (B) and Day (D) samples. Cyan arrowheads point to plaques at the tips of the microvilli. Orange rectangles in (A) and $(C)$ indicate the regions for which $3 \mathrm{D}$ volume renderings are shown in (B) and (D), respectively. White dotted lines in (D) indicate merged microvilli structures.

microvilli, each containing a single plaque at its tip, are merged to form anisotropic ridges around $300 \mathrm{~nm}$ in length (Figures 2C,D, 3D,E, and S1). The patterns observed in the microvilli structure and organization in Day and Night samples described here were consistent throughout different biological and technical replicates of the upper region of the distal part of hind tibia (see the Methods section) independent of the developmental stage of the analyzed animal.

To determine the microvilli organization over large areas (hundreds of microns), we prepared samples using chemical fixation methods and osmium staining followed by resin embedding. These samples are overstained and the images contain less details relative to cryofixed samples (Figure S2), facilitating automatic image analysis using two-dimensional neuronal networks (2D-FCN, see the Supporting Information for details). ${ }^{22}$ In both Day and Night samples, cell surface regions containing microvilli extend over several cells, but the spatial organization of the microvilli differs substantially between the two conditions (Figures S2 and 4C,D) in agreement with the results obtained from cryofixed samples. A comparison between the 3D Fourier transform (FT) of labeled volume of the microvilli in Day and Night datasets (Figure 4E-H) shows the differences in microvilli packing and organization. In Night samples, the organization is shortranged, whereas in Day samples, we observe second-order correlations indicating a longer range order. Furthermore, the arrangement is highly anisotropic in Day samples, where the distance $\left(d_{\mathrm{L}}\right)$ between the center of the ridges determined from the FT is $140 \mathrm{~nm}$ laterally and $250 \mathrm{~nm}$ vertically $\left(d_{\mathrm{V}}\right)$ but only slightly anisotropic in the Night samples $\left(d_{\mathrm{L}}=140 \mathrm{~nm}, d_{\mathrm{V}}\right.$ $=180 \mathrm{~nm}$ ). During the de novo formation of the embryo cuticle in Drosophila melanogaster, the apical membrane of the epidermal cells forms morphologically similar ridges, the apical undulae. However, in this case, they are elongated over several microns. $^{23}$

Orientation of Fibers in the Deposition Zone. The preservation of the assembly zone in cryofixed resin-embedded sections allowed determining the thickness and the orientation of the freshly deposited fibers in $3 \mathrm{D}$ within the assembly zone. Using FIB/SEM data, we determined fiber-bundle thickness of around $20 \mathrm{~nm}$. However, inspecting $100 \mathrm{~nm}$ thin sections using a transmitted electron detector (TED) in the FIB/SEM, we also observed thinner fibers around $5 \mathrm{~nm}$ (Figure S3). In both Day and Night samples, the fibers/fiber bundles assume parallel orientation with respect to the cuticle and the cell surface. The fibers/fiber bundles are stacked in the radial direction, with a slight lateral shift, giving the false appearance of oblique fibers protruding from the microvilli toward the cuticle when viewed in $2 \mathrm{D}$ in the (rt) plane (Figure S3). We speculate that the microvilli are dynamic structures that move laterally in the $(t z)$ plane while depositing the chitin fibers.

The fibers' long axes in the Day samples are parallel to the microvilli ridges' long axes (Figures $2 \mathrm{C}$ and $3 \mathrm{~F}$ ). In Night samples, we observe a narrow distribution of fiber orientation around three dominant orientations that seem to reflect the packing pattern of the microvilli (Figures $2 \mathrm{~A}$ and $3 \mathrm{C}$ ). In these samples, the dominant orientation of the fibers changes with the "depth" of the assembly zone, i.e., from the cell surface to the cuticle (Figure 5A,C), (with $\sim 50^{\circ}$ shift). We deduce from this observation that during the dark phase, the fibers are secreted to the assembly zone in discrete orientations (Figures $3 \mathrm{C}$ and $5 \mathrm{~A}, \mathrm{C})$. However, within the cuticle, the rotation angle between successive sheets is much smaller (in the order of $1^{\circ}$ ). This implies that the organization of the fibers to a helicoidal structure occurs via a self-assembly mechanism that includes reorientation and compactization of the fibers, where compactization refers to the process of fiber packing in the radial direction from the cell surface to the cuticle surface. In Day samples, as the fibers are deposited in their final orientation, compactization occurs without reorientation.

Microvilli Remodeling and the Appearance of Large Vesicles. In addition to the microvilli, in both Day and Night samples, we often observed large regions displaying different cell surface structures and a large number of vesicles (Figures 6 and S4-S6). These regions are at least as abundant as regions with microvilli. Occasionally, structures resembling the plaques in shape are present in these regions (Figure S6). This cell surface structure shows similarities to that found in kidney epithelial cells during remodeling of microvilli. ${ }^{24}$ The assembly 

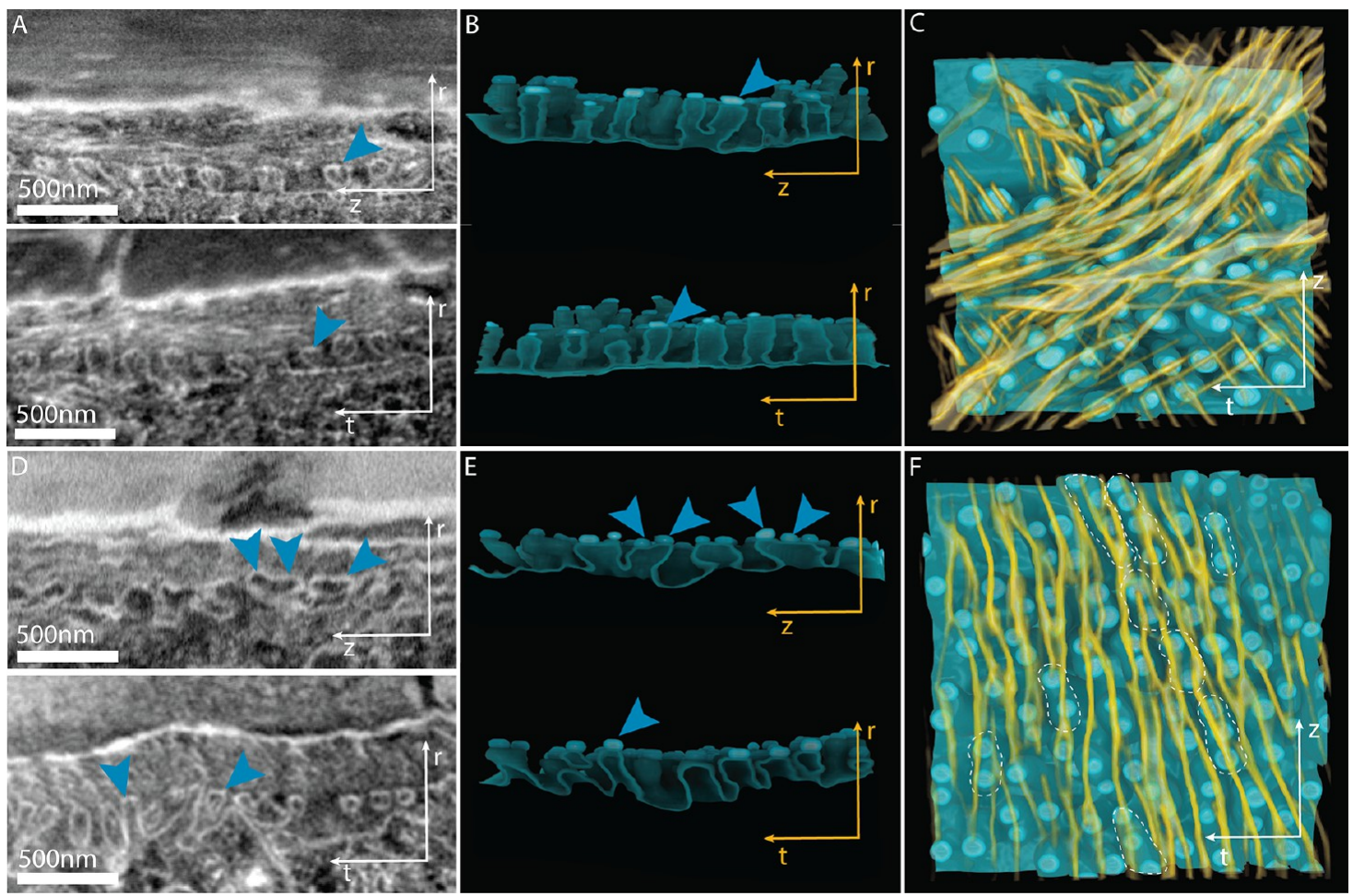

Figure 3. Microvilli structure and fiber deposition within the assembly zone. FIB/SEM images (A, D) obtained by reslicing the 3D datasets shown in Figure 2 along $(t)$ (top) and ( $z$ ) (bottom) directions for Night (A) and Day (D) samples. The comparison shows that the microvilli have similar dimensions in $(z)$ and $(t)$ directions in the Night samples ( $(\mathrm{A})$ top and bottom), whereas they are elongated along the $(z)$ direction in the Day samples ((D) top). The plaques are depicted in bright blue. (B, E) $(z r)$ and $(t r)$ plane views of resliced volumes. Only one plaque (bright blue arrowhead) is situated at the tips of each microvillus in Night samples (B), but two or three plaques are observed in the Day samples on top of microvilli merged along the $(z)$ direction $(\mathrm{E})$. (C, F) Three-dimensional volume rendering of the chitin fibers/fiber bundles (yellow) observed in the assembly zone and the apical cell surface in Night (C) and Day (F) samples. For simplicity, only half of the assembly zone thickness is shown. White dotted lines in (F) indicate merged microvilli structures.

zone at this stage is narrower and highly stained such that the fibers cannot be discriminated (Figure S3B). We speculate that this stage may be related to vesicular protein secretion and potentially to the remodeling of the microvilli. Thus, our observations suggest an active cell surface with a temporal process with at least two timescales, one in which dynamic microvilli move laterally while depositing chitin fibers and a second stage in which the microvilli disappear and are replaced by vesicles.

Three-dimensional FIB/SEM is an excellent tool to characterize mesoscale structures like the apical cell surface and the fiber orientation; however, this technique is insensitive to the molecular structure and organization of the chitin fiber and associated proteins. To gain further insight into the chitin and protein molecular assemblies, we used scanning X-ray scattering. Hind tibia of animals reared in $12 / 12 \mathrm{~h}$ illumination cycle were rapidly frozen and thin sections $(\sim 30 \mu \mathrm{m})$ were prepared using a cryo-microtome. The sections were air-dried and mounted on X-ray transparent silicon nitride membranes. The sections were scanned using a monochromatic focused Xray beam (beam diameter $\sim 1 \mu \mathrm{m}$ ), while $2 \mathrm{D}$ X-ray diffraction (XRD) patterns and X-ray fluorescence (XRF) were recorded concomitantly at each position. We used the (110) chitin reflection to localize the cuticle and the $\mathrm{Zn} \mathrm{XRF} \mathrm{signal} \mathrm{for} \mathrm{the}$ localization of the cells. The intersection of the two signals marks the location of the assembly zone (Figure S7).

Fiber Compactization. Small-angle X-ray scattering (SAXS) provides information about the spatial nanoscale organization of the chitin-protein fibers within the assembly zone and within the cuticle in the section plane. The SAXS intensity was integrated radially and plotted as a function of the azimuth angle (Figure S8). The width of the peaks in this plot is related to the degree of fiber alignment with respect to each other within the $(r t)$ plane (as defined in Figure 1A). The SAXS peak width (full width at half-maximum (FWHM), in units of degrees, Figure S8) is mapped in Figure S7B,D across a tibia cross section in Night and Day samples, respectively. In both cases, the SAXS peak width at the assembly zone is broader than the peak width in regions within the cuticle bulk. This is supportive of a compactization process occurring after deposition as discussed above.

Chitin-Protein Co-assembly. X-ray diffraction (XRD) provides information about the molecular structure, the respective organization, and the interaction of chitin with the cuticular proteins. ${ }^{25,26} \mathrm{We}$ have recently shown that coordering of chitin and proteins along the $b$-crystallographic direction of chitin, in the tarsal-tendon of the spider Cupiennius salei, gives rise to a shift in the (020) chitin reflection due to coherent scattering. ${ }^{26}$ In addition, protein-specific reflections with d-spacing equal to $0.47 \mathrm{~nm}$, characteristic of intrasheet interstrand spacing in cross $\beta$-sheet motifs, were observed along the meridian in $2 \mathrm{D}$ fiber diffraction patterns of spider tendons. To best identify protein-related reflections in the locust tibia, we reared animals in $24 \mathrm{~h}$ light settings. In these conditions, the endocuticle is made only of unidirectional layers. ${ }^{19}$ The fiber diffraction profiles obtained from longi- 

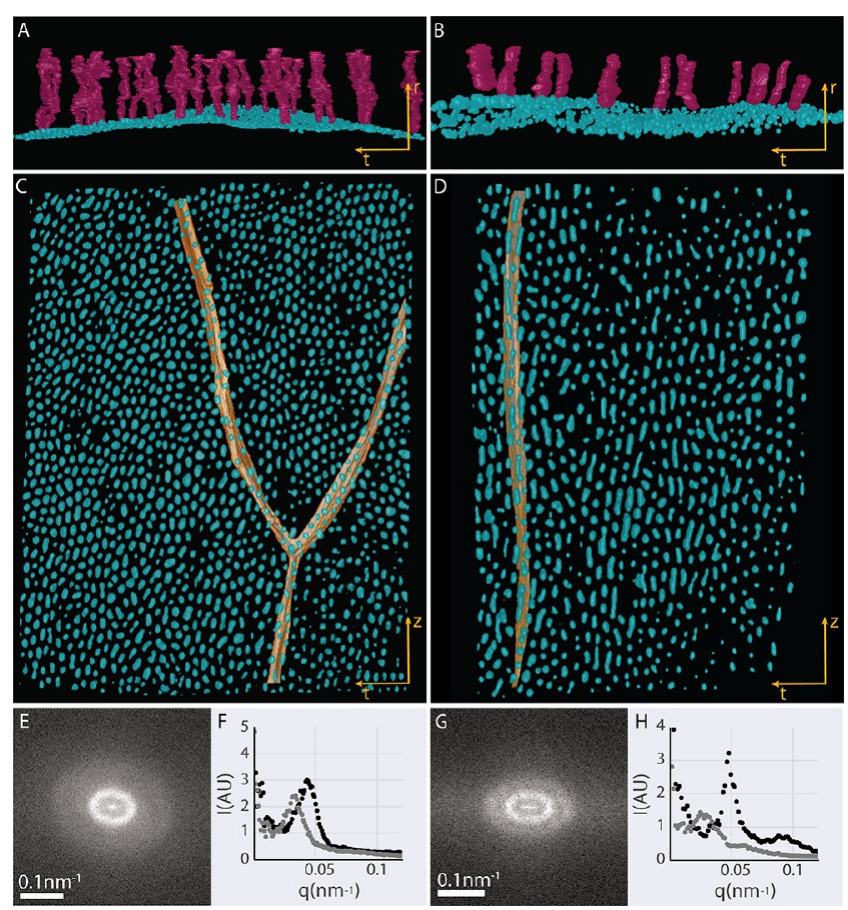

Figure 4. Reconstruction and quantification of 3D FIB/SEM data of apical cell surface structures obtained from chemically fixed locust tibiae. (A, B) Volume rendering of the segmented apical protrusions at the surface of the epidermal cells (cyan) and the pore canals (magenta) in Night (A) and Day (B) samples of fifth instar animals 2 days after ecdysis. (C, D) "Top-view" of the rendered volume of the segmented apical surface of the epidermal cells (cyan) and the lateral cell membrane (orange) in Night (C) and Day (D) samples. Note that the respective apical protrusion organization is continuous across multiple cells. (E, G) Three-dimensional Fourier transform (FT) of the segmented volumes representing the epidermal cell surfaces in Night (E) and Day (G) samples. (F, H) Azimuthal partial integration of the FT pattern in (E) and (F), respectively, in the meridional (light gray) and equatorial (dark gray) directions, showing increased isotropy and reduced long-range-order correlation of the apical protrusions in Night vs Day samples.

tudinal sections $(\sim 70 \mu \mathrm{m}$ thick $)$ of such samples show a shift in the (020) reflection of chitin as well as several proteinrelated reflections along the meridian at $d=0.47 \mathrm{~nm}$ (as observed in the spider tendon diffraction pattern) and at $d=$ $0.43 \mathrm{~nm}$ (Figure S9). Interstrand spacing of $0.47 \mathrm{~nm}$ is common for $\beta$-sheet structures as in amyloids, whereas spacing of $0.43 \mathrm{~nm}$ is common for the interstrand spacing in silks. ${ }^{27-29}$ Other unassigned reflections with $d$-spacings of 0.38 and 0.77 $\mathrm{nm}$ are observed along with additional reflections in the smallangle region with $d$-spacings of 1.5 and $3.4 \mathrm{~nm}$ (the latter already noted by Rudall et al. for the cuticle of various insect species $^{30}$ ). Based on these results, we suggest that at least a large portion of the cuticular proteins contains cross- $\beta$ structures, where the $\beta$-strand orientation is roughly perpendicular to the chitin fiber long axis in unidirectional cuticle. Although individual protein reflections are not visible in helicoidal cuticles due to their low intensity and overlap with chitin reflections, the presence of the ordered proteins is evident by apparent peak broadening of the chitin reflections as well as the shift in the (020) reflection.

Based on the structural information gained from unidirectional oriented cuticle, we used scanning X-ray diffraction to study the local chitin-protein molecular organization within
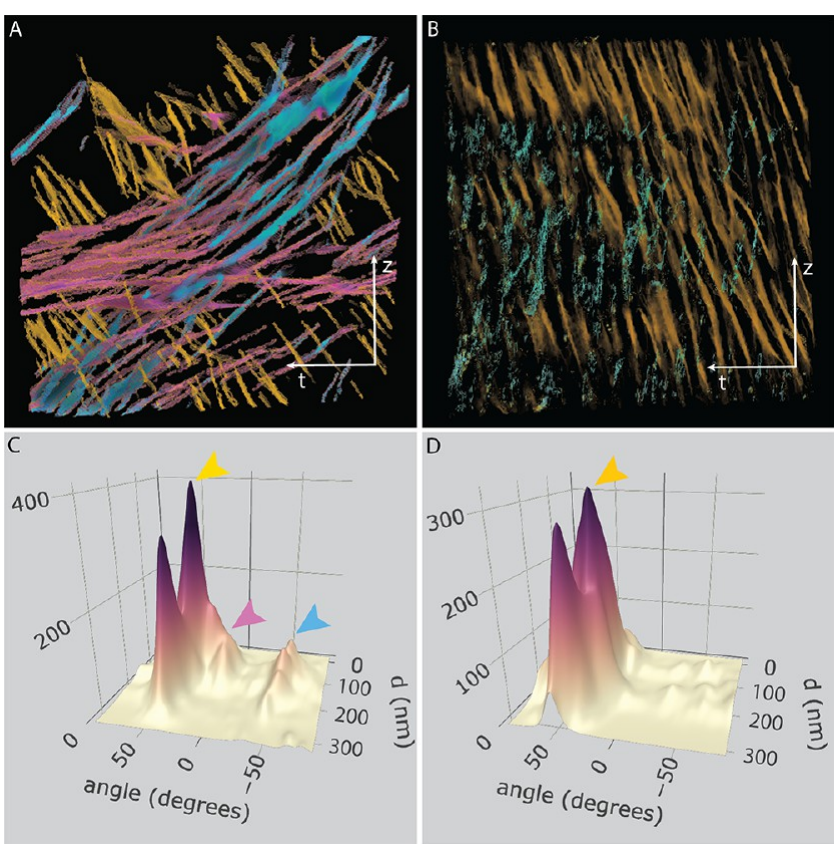

Figure 5. Fiber orientation in the assembly zone. Orientation color maps of the fibers in the assembly zone (entire volume) in Night (A) and Day (B) samples from datasets shown in Figures 2 and 3. (C, D) Fiber orientation (angle) vs assembly zone depth $(d, \mathrm{~nm})$ histograms showing the variation in fiber orientation as a function of their position within the assembly zone along the $(r)$ direction from the cell surface $(0 \mathrm{~nm})$ to the cuticle $(300 \mathrm{~nm})$. The color (yellow, pink, blue, orange) arrowheads represent the respective dominant orientations in (A) and (B).

different cuticular layers as well as within the assembly zone. Here, we used cross sections of the tibia, such that the fiber orientation in unidirectional layers is parallel to the X-ray beam (similar to the SAXS experiment above). In this orientation, we identify an additional protein reflection with a $d$-spacing of 1.1 $\pm 0.05 \mathrm{~nm}\left(q=5.5 \mathrm{~nm}^{-1}\right)$. Reflections at these positions are often assigned to intersheet spacing in stacking $\beta$ sheets when they are accompanied with orthogonal intrasheet reflections such as the ones observed here $(d=0.47 \mathrm{~nm})$. This result is in good agreement with the previous prediction of a double layer of cross $\beta$-sheet stacking. ${ }^{26}$ Note that according to the theory, ${ }^{26}$ when coherent chitin/protein scattering occurs, a separate protein reflection is unexpected. That this reflection is observed suggests that not all of the proteins share a coherent interface with the chitin crystallites and/or that coherence occurs only along the chitin $b$-direction, while the proteins surround the chitin in all directions in the $a b$ plane.

The (020) reflection of chitin and the protein intersheet reflection were peak-fitted for each diffraction profile within the scanned region. Mapping the intensity of the (020) peak (Figure 7A) allows assigning regions of unidirectional organization (high peak intensity) produced during the day vs regions of helicoidal organization (low peak intensity) produced at night. The peak position of the $(020)$ reflection is shifted in the cuticle to lower $q$ values $\left(q \sim 6.35 \mathrm{~nm}^{-1}\right)$, with respect to pure chitin $\left(q=6.61 \mathrm{~nm}^{-1}\right)$, as seen before in samples from locusts grown in all-day conditions. In the assembly zone, however, this reflection is closer $(q \sim 6.5$ $\mathrm{nm}^{-1}$ ) to pure chitin (Figure $7 \mathrm{C}$ ), suggesting that in this region the proteins and chitin are not co-ordered to produce a coherent diffraction interference. A map of the chitin to 

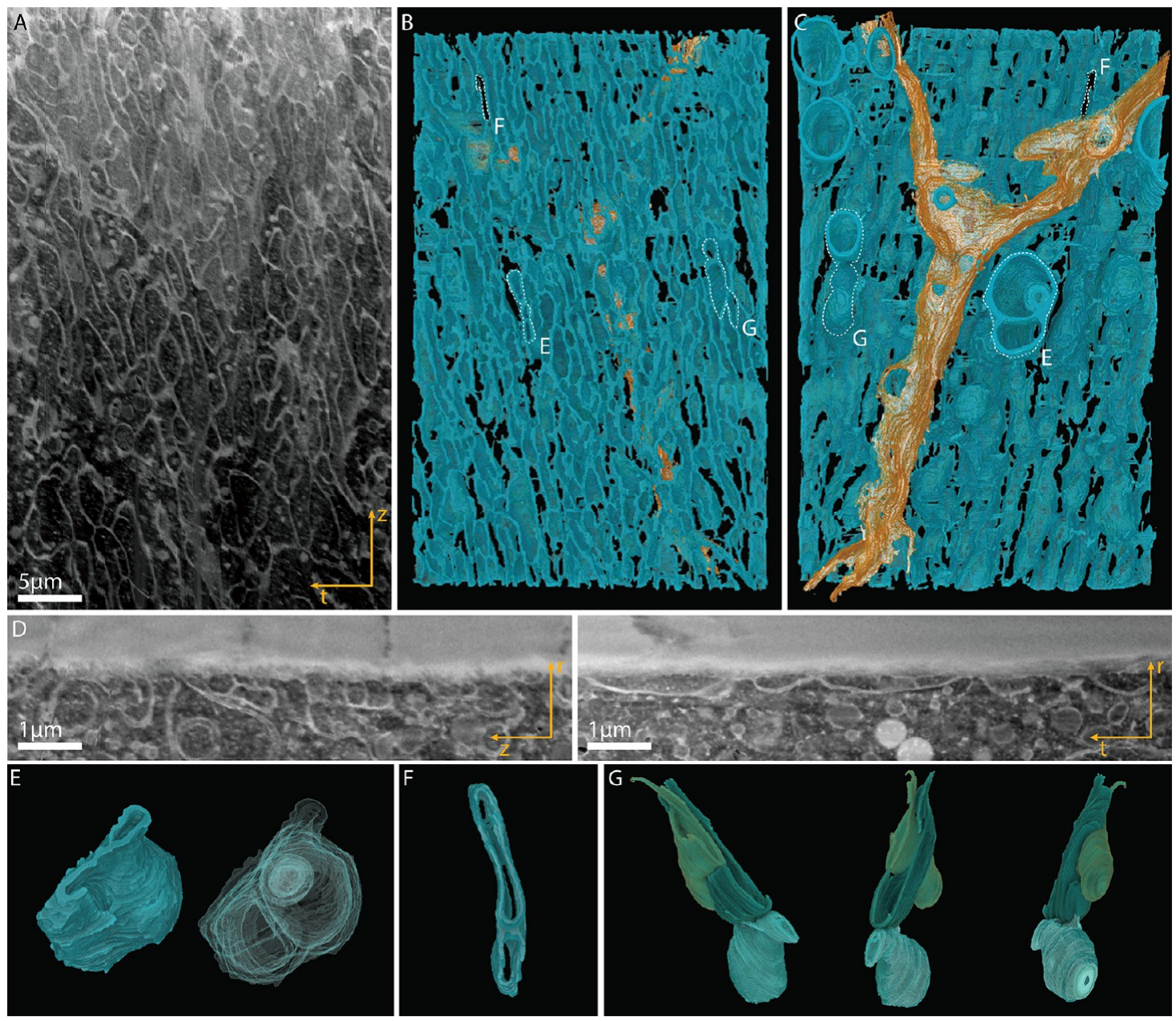

Figure 6. Apical surface of the epidermal cells in the absence of microvilli. (A) FIB/SEM slice at the cell surface showing irregular structures in a Day sample of fifth instar animal 2 days after ecdysis. (B, C) Volume rendering of the 3D reconstructed FIB/SEM data represented in (A) in the $(t z)$ plane. In (B), the cell surface is viewed from the outside of the cell. In (C), the cell surface is viewed from within the cell outward. Lateral cell membranes are depicted in orange. Individual structures depicted in $(E)-(G)$ are marked with a white dashed line in both (B) and (C). (D) Three-dimensional FIB/SEM data resliced to show the $(t r)$ and $(z r)$ plane views. (E-G) Three-dimensional volume rendering of representative structures visible in (B) and (C). (E) Multivesicular body. (F) Elongated openings at the cell surface. (G) Vesicles presumably fusing with the apical cell membrane.
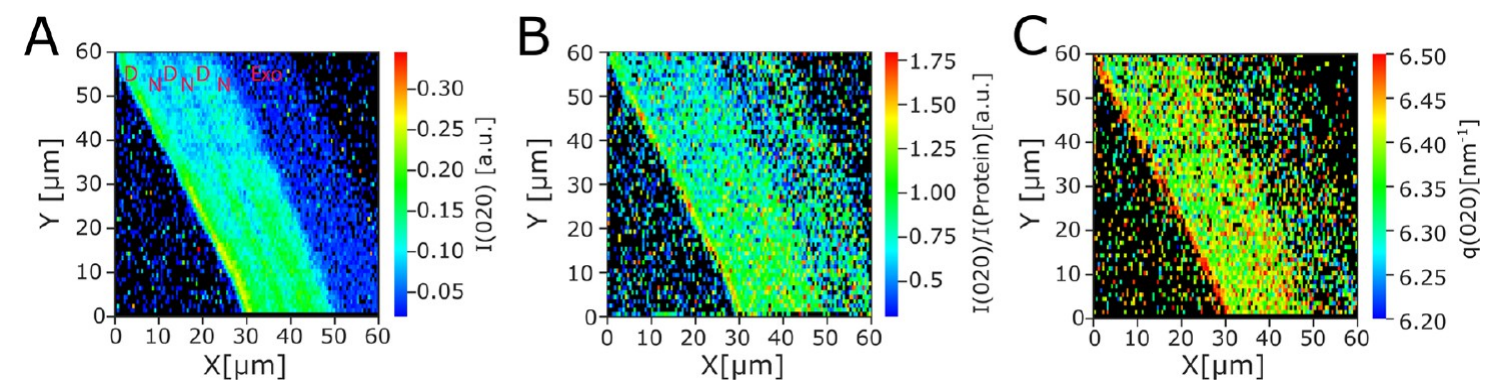

Figure 7. Scanning X-ray diffraction measurements of adult locust tibia cross sections 4 days after ecdysis. (A) Intensity variation of the (020) reflection allows identification of "Day" (high $\left.I_{(020)}\right)$ and "Night" (low $\left.I_{(020)}\right)$ regions. Exo marks the region of exocuticle, D marks Day regions, and $\mathrm{N}$ marks Night regions. (B) Integrated intensity ratio between the chitin reflection $(020)$ and the protein reflection $\left(q \sim 5.5 \mathrm{~nm}^{-1}\right)$. The ratio is increased in the assembly zone relative to the cuticle. (C) Peak position $(q)$ of the $(020)$ reflection. The $q(020)$ position is shifted in the cuticle relative to the assembly zone.

protein ratio based on the integrated intensity of the protein peak at $5.5 \mathrm{~nm}^{-1}$ and the (020) peak (Figure $7 \mathrm{~B}$ ), as well as the azimuthal integration of $2 \mathrm{D}$ patterns taken from the assembly zone (Figure S10), show that the intersheet protein peak has lower intensity in the assembly zone relative to the cuticle (in both unidirectional and helicoidal regions).
Increased protein diffraction in the cuticle accompanied with the (020) peak shift (Figure 7) suggests chitin-protein coassembly. Thus, compactization, as observed from SAXS analysis, and co-alignment of chitin and protein, as judged from the XRD analysis, are co-localized, implying that coassembly of the protein and chitin is the driving force for fiber 
reorientation. We note that the phase diagram of chitin in water is extensively explored and well characterized, including the helicoidal pitch dependence on $\mathrm{pH}$, ionic strength, and crystallite size. ${ }^{31-33}$ Unfortunately, however, a direct correlation of our findings with the lyotropic behavior of chitin as determined from in vitro experiments in aqueous solutions is limited as the physicochemical conditions in the assembly zone are to date unknown and as the effect of chitin-binding proteins on chitin assembly has not been systematically addressed experimentally in vitro.

In summary, our results suggest that dynamic apical cell surface structures (microvilli) in the cuticular epidermal layer determine the boundary conditions for fiber self-assembly by secreting the fibers into the assembly zone in predetermined orientations. During the night, when a helicoidal fiber arrangement is formed, the fiber orientations in the assembly zone are discrete, mirroring the microvilli spatial organization, whereas when parallel fiber arrangement is produced during the day, the fiber orientation in the assembly zone is parallel to the elongated microvilli ridge structures. The final orientation of the fibers is achieved by concomitant chitin-protein coordering and compactization of the cuticle, resulting in a stable helicoidal or unidirectional structure in the endocuticle. We note that the helicoidal organization could, theoretically, be produced by employing a similar cell surface structure as seen in light conditions and rotating the orientation of the ridges between deposition cycles to produce the rotated plywood structure. This is however not the case, since the apical cell surface structures differ significantly in microvilli morphology between light and dark conditions. This leads us to conclude varying levels of cellular control on the assembly process in line with Neville's two-model system suggestion. ${ }^{18}$ Here, the unidirectional arrangement is under direct cellular control on fiber orientation, whereas the helicoidal structure is obtained away from the apical cell surface by self-assembly. Indeed, the aligned nematic organization of chitin is also unachievable in vitro without the application of external force. ${ }^{16}$ Based on our $\mathrm{X}$-ray data, we propose that chitin-protein co-assembly and protein-protein interactions are the driving forces for the process. Our results provide direct evidence for the selfassembly hypothesis put forward by Neville, ${ }^{18}$ Giraud-Guille, and Bouligand ${ }^{17,34}$ based on the architecture of the cuticle in its final form and its resemblance to liquid crystal geometries observed in vitro. The observations and the methodology presented here could be relevant for a variety of biological extracellular matrices where self-assembly and cell-controlled mechanistic questions are yet unresolved.

\section{METHODS}

First, fourth, and fifth instar specimen of L. migratoria were obtained from Reptilienkosmos (www.reptilienkosmos.de). The animals were reared in $12 / 12 \mathrm{~h}$ day/night cycle or in $24 \mathrm{~h}$ daylight and 36/26 or $36{ }^{\circ} \mathrm{C}$ conditions, respectively. The light source was a Mini light strip light-emitting diode (LED) (Lucky Reptile) emitting a daylight spectrum at $200 \mathrm{~lm}$. Day samples were obtained during the light phase of the illumination cycle, whereas Night samples were obtained during its dark phase.

FIB/SEM Sample Preparation. Chemical Fixation. Two biological replicates of hind tibia of both fifth instar and adult $L$. migratoria were collected during the light and the dark phase of the illumination cycle 2 days after ecdysis. The entire hind tibia was submerged in $2.5 \%(\mathrm{w} / \mathrm{v})$ glutaraldehyde and $2 \%(\mathrm{w} / \mathrm{v})$ paraformaldehyde in 0.1 $\mathrm{M}$ cacodylate buffer $(\mathrm{pH} 7.4)$ and incubated for $4 \mathrm{~h}$ at room temperature. Samples were washed five times for $10 \mathrm{~min}$ in
$0.1 \mathrm{M}$ cacodylate buffer and incubated for $2 \mathrm{~h}$ in $2 \%(\mathrm{w} / \mathrm{v})$ osmium tetroxide. The samples were washed again in $0.1 \mathrm{M}$ cacodylate buffer five times for $10 \mathrm{~min}$ each and dehydrated in a series of ascending concentrations of acetone $(30,50,70,90,100 \% 2 \times)$ with $10 \mathrm{~min}$ for each step. Tissues were then infiltrated with Durcupan/Epon epoxy $\operatorname{resin}^{35}$ and cured at $60{ }^{\circ} \mathrm{C}$ for $48 \mathrm{~h}$. Cross sections of the upper region of distal part of hind tibia (see Figure 1A) were cut, polished (sandpaper (Grit 2000)), and coated with $5 \mathrm{~nm}$ carbon and $5 \mathrm{~nm}$ platinum and imaged using focused an ion beam/scanning electron microscope (FIB/SEM). In total, we acquired two Day datasets from two fifth instar animals, two Day datasets from two adult stage animals, four Night datasets from two fifth instar animals, and three Night datasets from two adult stage animals.

Cryofixation. Two or three biological replicates of hind tibia of fourth and fifth instar and adult L. migratoria and one biological replicate of third instar were collected during the light and the dark photoperiod 2 or 3 days after ecdysis. For the fifth instar and adult specimens, we used a sharp razor blade to manually trim the upper region of the distal part of tibia (Figure 1A) into 100-200 $\mu \mathrm{m}$ thick cross sections. The thickness of the whole tibia of the fourth and third instar specimens was below $200 \mu \mathrm{m}$. We therefore trimmed the length of the tibia to fit the sample carriers using a razor blade. Throughout this time, the samples were submerged in standard locust saline solution. ${ }^{36}$ The samples were immediately vitrified using a highpressure freezing machine (HM100, Leica). Briefly, two sections were loaded into a cavity of a single B-type carrier and $20 \%$ dextran in standard locust saline solution was used as a filler and cryoprotectant. Samples were covered with a flat side of a second B-type carrier. Vitrified samples were freeze-substituted with a fixation and staining cocktail ( $1 \%$ osmium tetroxide, $0.1 \%$ uranyl acetate, $0.5 \%$ glutaraldehyde, $1.5 \%$ water) in acetone over 2 days at $-85{ }^{\circ} \mathrm{C}$ using an automatic freeze-substitution machine (AFS2, Leica). The samples were brought to room temperature over 1 day and embedded as described above. Polymerized resin blocks were further polished using a fine sandpaper (Grit 2000) to expose the cross sections for block-surface imaging. After coating the surface with carbon and platinum as described above, samples were imaged using FIB/SEM. In total, we acquired 1 Day dataset from 1 third instar animal, 2 Day datasets from 2 fourth instar animals, 7 Day datasets from 3 fifth instar animals, 10 Day datasets from 3 adult specimens, 1 Night dataset from 1 third instar animal, 2 Night datasets from 2 fourth instar animals, 6 Night datasets from 3 fifth instar animals, and 3 Night datasets from 2 adult specimens. The results and the trends observed in each of the samples were consistent with the main observations reported in the Results and Discussion section.

In chemically fixed samples, the formed fibers in the assembly zone cannot be resolved due to excessive cross-linking leading to overstaining. We therefore used the geometry of the pore canals (Figures S2 and 5) to determine the fiber orientation in the last deposited layers: the pore canals are channels running throughout the cuticle containing cellular processes characterized by their almond shape cross section. When the pore canals pass through the lamellate helicoidal cuticle, the cross section shows a continuous rotation, which is absent in the nonlamellate unidirectional cuticle architecture. $^{18}$

FIB/SEM Image Serial Imaging (Crossbeam 540, Zeiss). For chemically fixed datasets representing Night samples, 538 serial electron micrographs were acquired in $r t$ plane with a voxel size $(13.55 \times 13.55 \times 17.5) \mathrm{nm}^{3}$. For dataset representing chemically fixed Day samples, 393 serial electron micrographs were acquired in $r t$ plane with a voxel size $(12.82 \times 12.82 \times 17.5) \mathrm{nm}^{3}$. In both cases, SEM imaging was performed at $2 \mathrm{kV}$ acceleration voltage and $1 \mathrm{nA}$ probe current using secondary electron detector, while slices were generated using the $300 \mathrm{pA}$ FIB probe at a $30 \mathrm{kV}$ accelerating voltage.

For dataset representing cryofixed samples, the number of slices ranged from around 800 to 2270 and voxel size from $(5.28 \times 5.28 \times$ $10.5) \mathrm{nm}^{3}$ to $(8.1 \times 8.1 \times 21) \mathrm{nm}^{3}$. In all cases, SEM imaging was performed at $2 \mathrm{kV}$ acceleration voltage and $1 \mathrm{nA}$ probe current using secondary electron detector, while slices were generated using the 300 pA FIB probe at a $30 \mathrm{kV}$ accelerating voltage. 
Transmission Electron Detector (TED) Imaging. TED imaging was performed inside the FIB/SEM chamber using a STEM4A detector. Thin sections $(100 \mathrm{~nm})$ of cryofixed samples were prepared using a Leica UC- 6 microtome. The sections were mounted on copper grids and imaged with a $20 \mathrm{kV}$ acceleration voltage and 300 pA probe current using high-resolution column mode.

FIB/SEM Image Analysis. Image stacks were aligned, cleared for curtaining artifacts, ${ }^{37}$ and denoised using the scikit-image python library (v.0.14.0). Segmentation was performed semiautomatically using a combination of Amira 3D (FEI) and a custom python code implementing state of the art $2 \mathrm{D}$ and $3 \mathrm{D}$ machine learning methods using Keras and GPU accelerated Tensorflow (see details in the Supporting Information).

The Drishti volume exploration and representation tool (v.2.6.5) was used for volume rendering. ${ }^{38}$ Orientation maps and distributions were generated with Orientation J plugin of Fiji (http://bigwww.epfl. $\mathrm{ch} /$ demo/orientation/). Three-dimensional FTs of the stack of the labeled microvilli were calculated using scipy (v.1.3.0). Azimuthal averages of the $2 \mathrm{D}$ FTs were calculated using a custom script in python.

SAXS/Wide-Angle X-ray Scattering (WAXS) Sample Preparation. The distal tibia parts of L. migratoria adult animals reared in $12 \mathrm{~h}$ dark $/ 12 \mathrm{~h}$ light cycle or $24 \mathrm{~h}$ light were severed from the animals 3, 5 or 14 days after ecdysis, respectively. Specimens were embedded in O.C.T. (VWR Chemicals, Radnor, PA) and rapidly frozen using liquid nitrogen in a silicon mold. The frozen blocks were sectioned using a HM 560 CryoStar Cryostat (Thermo Fisher Scientific, Waltham, MA) with Surgipath DB80 LX blades (Leica, Wetzlar, Germany) (sample $-15{ }^{\circ} \mathrm{C}$, blade $-11{ }^{\circ} \mathrm{C}$ ). For the $12 \mathrm{~h}$ dark $/ 12 \mathrm{~h}$ light samples, cross sections of 20 and $30 \mu \mathrm{m}$ thickness were prepared for SAXS and WAXS measurements, respectively. In the case of the 24 h light samples, longitudinal sections of $70 \mu \mathrm{m}$ thickness were cut. The sections were thawed, rinsed with water, and transferred to a $\mathrm{Si}_{3} \mathrm{~N}_{4}$ membrane (Silson, Southam, U.K.) $(3 \times 3$ window array (each $(5 \times 5) \mathrm{mm}^{2}, 1 \mu \mathrm{m}$ thick $)$, frame thickness $200 \mu \mathrm{m},(23.5 \times 23.5)$ $\mathrm{mm}^{2}$ total size) for XRD/XRF measurement.

SAXS/XRD and XRF Mapping. Simultaneous X-ray fluorescence and scattering experiments were performed at the microXAS-X05LA beamline at the Swiss Light Source (SLS) synchrotron radiation facility (Paul Scherrer Institute, Villigen, Switzerland). The X-ray beam was defined to $15.2 \mathrm{keV}(0.816 \AA)$ using a $\mathrm{Si}(111)$ monochromator and focused to $(1 \times 1) \mu \mathrm{m}^{2}$ using $\mathrm{KB}$ mirrors. XRD data were obtained using a $2 \mathrm{D}$ Dectris Eiger $9 \mathrm{M}$ detector $\left((2070 \times 2167)\right.$ pixel $\left.^{2}\right)$ in transmission geometry, and a singleelement $\mathrm{Si}(\mathrm{Li})$ XRF detector (Ketek, Munich, Germany) was placed perpendicular to the beam. XRD data from $24 \mathrm{~h}$ day sample were obtained at the $\mu$ Spot beamline at the synchrotron BESSY II (Helmholtz Center, Berlin, Germany). The X-ray energy of $15 \mathrm{keV}$ $(0.827 \AA)$ was defined by a multilayered monochromator. The incident X-ray beam was defined by a toroidal mirror and a pinhole of $10 \mu \mathrm{m}$. WAXS data were collected using a large-area 2D detector (MarMosaic 225, Mar USA Evanston).

Calibration, integration background removal, and peak-fitting of the $2 \mathrm{D}$ diffraction patterns were performed using the software DPDAK. $^{39}$ For plotting the data, OriginPro 2015 software and matplotlib library in Spyder 3.3.2 from the Anaconda package were used. ${ }^{40}$

Confocal Laser Scanning Microscopy (CLSM). Sections were fixed and sectioned as described above using a cryo-microtome. They were stained with Nile red and DAPI or with Direct Yellow 96 (Sigma-Aldrich) in standard locust saline solution for $1 \mathrm{~h}$. Images were acquired using a SP-8 laser scanning confocal microscope (Leica) equipped with a $63 \times$ water immersion objective $(\mathrm{NA}=1.2)$. A multiphoton laser with a wavelength of $780 \mathrm{~nm}$ was used for the excitation of DAPI and Direct Yellow 96, and the signal was recorded by a HyD detector with a bandpass filter set to $400-480$ and 540$610 \mathrm{~nm}$, respectively. For Nile red, a DPS S 561 laser emitting light of $561 \mathrm{~nm}$ was used for excitation together with a $\mathrm{HyD}$ detector with a bandpass filter of $570-620 \mathrm{~nm}$ for detection of the fluorescence signal.

\section{ASSOCIATED CONTENT}

Supporting Information

The Supporting Information is available free of charge at https://pubs.acs.org/doi/10.1021/acsami.0c04572.

FIB/SEM slices of cryofixed locust tibiae (Figure S1); FIB/SEM slices of chemically fixed locust tibiae (Figure S2); TED micrographs (Figure S3); FIB/SEM slice series from regions in a Day sample from a fifth instar animal 2 days after ecdysis lacking microvilli (Figure S4); segmentation of FIB/SEM data (Figure S5); FIB/ SEM data of epidermal cell apical surfaces in adult specimen and fifth instar sampled 2 days after ecdysis (Figure S6); scanning X-ray scattering and fluorescence results (Figure S7); SAXS peak azimuthal width (Figure S8); WAXS data of adult locust tibia (Figure S9); averaged azimuthally integrated X-ray diffraction data (Figure S10) (PDF)

\section{AUTHOR INFORMATION}

\section{Corresponding Authors}

Luca Bertinetti - Department of Biomaterials, Max Planck Institute of Colloids and Interfaces, 14476 Potsdam, Germany; ○ orcid.org/0000-0002-4666-9610; Email: luca.bertinetti@ mpikg.mpg.de

Yael Politi - Department of Biomaterials, Max Planck Institute of Colloids and Interfaces, 14476 Potsdam, Germany; ำ orcid.org/0000-0002-2872-8277; Email: yael.politi@ mpikg.mpg.de

\section{Authors}

Sanja Sviben - Department of Biomaterials, Max Planck Institute of Colloids and Interfaces, 14476 Potsdam, Germany

Oliver Spaeker - Department of Biomaterials, Max Planck Institute of Colloids and Interfaces, 14476 Potsdam, Germany

Mathieu Bennet - Department of Biomaterials, Max Planck Institute of Colloids and Interfaces, 14476 Potsdam, Germany

Marie Albéric - Department of Biomaterials, Max Planck Institute of Colloids and Interfaces, 14476 Potsdam, Germany; Laboratoire Chimie de la Matiere Condensée de Paris, Sorbonne Université, UMR CNRS 7574, 75005 Paris, France; (1) orcid.org/0000-0002-5322-9904

Jan-Henning Dirks - Max Planck Institute for Intelligent Systems, 70569 Stuttgart, Germany; Biomimetics-InnovationCentre, Hochschule Bremen-City University of Applied Sciences, 28199 Bremen, Germany

Bernard Moussian - Institute of Biology Valrose, Universite Côte d'Azur, CNRS, Inserm, 06108 Nice, France

Peter Fratzl - Department of Biomaterials, Max Planck Institute of Colloids and Interfaces, 14476 Potsdam, Germany

Complete contact information is available at: https://pubs.acs.org/10.1021/acsami.0c04572

\section{Author Contributions}

${ }^{\#}$ O.S. and M.B. contributed equally to this work.

\section{Funding}

We are grateful to the Deutsche Forschungsgemeinschaft (DFG) for financial support within the project DI2015/2-1.

\section{Notes}

The authors declare no competing financial interest. All data, code, and materials used are available upon request. 


\section{ACKNOWLEDGMENTS}

We appreciate the help of Birgit Schonert from the Max Planck Institute of Colloids and Interfaces with sample preparation. We thank Prof. Thomas Stach (Humboldt University, Berlin), Drs. Katja Höflich and Holger Kropf (Helmholtz-Zentrum Berlin für Materialien und Energie, Berlin, Germany), and Dr. Igor Zlotnikov (B CUBE, Technical University Dresden, Dresden) for assistance with TEM and FIB/SEM imaging. Thank you to Sebastian Wieser, Lisa Schoop, and Romy Angermann for help with animal maintenance and sampling. We are grateful to Drs. Changhao Li and Stefan Siegel for beamtime assistance during the measurements at BESSY II, Helmholtz-Zentrum Berlin (HZB), Germany, and to Drs. Grolimund Daniel and Dario Ferreira Sanchez from the Swiss Light Source, Paul Scherrer Institute (PSI), Villigen, Switzerland, for invaluable assistance during beamtime at the microXAS-X05LA beamline. We acknowledge HZB, Germany, and PSI, Villigen, Switzerland, for the allocation of synchrotron radiation beamtime at beamlines $\mu$ Spot at Bessy II and microXAS-X05LA of the SLS, respectively. A special thank you is given to Prof. Friedrich Barth, University of Vienna, Vienna, Austria, Prof. Nadine Nassif, Sorbonne Universités, CNRS, Paris, France, and Dr. Yoshiharu Nishiyama, University Grenoble Alpes, CNRS, CERMAV, Grenoble, France, for fruitful and inspiring discussions. Credits for the L. migratoria picture in Figure ${ }^{1} \mathrm{~A}$ go to AIDAsign-stock.adobe.com.

\section{REFERENCES}

(1) Dunlop, J. W. C. C.; Fratzl, P. Biological Composites. Annu. Rev. Mater. Res. 2010, 40, 1-24.

(2) Bouligand, Y. Twisted Fibrous Arrangements in Biological Materials and Cholesteric Mesophases. Tissue Cell 1972, 4, 189-217.

(3) Neville, A. C. Biology of Fibrous Composites: Development beyond the Cell Membrane; Cambridge University Press: New York, NY, 1993.

(4) Giraud-Guille, M. M.; Mosser, G.; Belamie, E. Liquid Crystallinity in Collagen Systems in Vitro and in Vivo. Curr. Opin. Colloid Interface Sci. 2008, 13, 303-313.

(5) Mitov, M. Cholesteric Liquid Crystals in Living Matter. Soft Matter 2017, 13, 4176-4209.

(6) Livolant, F.; Bouligand, Y. Liquid Crystalline Phases given by Helical Biological Polymers (DNA, PBLG and Xanthan). Columnar Textures. J. Phys. 1986, 47, 1813-1827.

(7) Neville, A. C. Chitin Orientation in Cuticle and Its Control. Advances in Insect Physiology; Academic Press, 1967; Vol. 4, pp 213286.

(8) Belamie, E.; Mosser, G.; Gobeaux, F.; Giraud-Guille, M. M. Possible Transient Liquid Crystal Phase during the Laying out of Connective Tissues: $\alpha$-Chitin and Collagen as Models. J. Phys. Condens. Matter 2006, 18, S115-S129.

(9) Yu, R.; Liu, W.; Li, D.; Zhao, X.; Ding, G.; Zhang, M.; Ma, E.; Zhu, K. Y.; Li, S.; Moussian, B.; et al. Helicoidal Organization of Chitin in the Cuticle of the Migratory Locust Requires the Function of the Chitin Deacetylase2 Enzyme (LmCDA2). J. Biol. Chem. 2016, 291, 24352-24363.

(10) Luschnig, S.; Bätz, T.; Armbruster, K.; Krasnow, M. A. Serpentine and Vermiform Encode Matrix Proteins with Chitin Binding and Deacetylation Domains That Limit Tracheal Tube Length in Drosophila. Curr. Biol. 2006, 16, 186-194.

(11) Moussian, B.; Tång, E.; Tonning, A.; Helms, S.; Schwarz, H.; Nüsslein, C.; Uv, A. E. Drosophila Knickkopf and Retroactive Are Needed for Epithelial Tube Growth and Cuticle Differentiation through Their Specific Requirement for Chitin Filament Organization. Development 2004, 163-171.

(12) Petkau, G.; Wingen, C.; Jussen, L. C. A.; Radtke, T.; Behr, M. Obstructor-A Is Required for Epithelial Extracellular Matrix
Dynamics, Exoskeleton Function, and Tubulogenesis. J. Biol. Chem. 2012, 287, 21396-21405.

(13) Noh, M. Y.; Kramer, K. J.; Muthukrishnan, S.; Kanost, M. R.; Beeman, R. W.; Arakane, Y. Two Major Cuticular Proteins Are Required for Assembly of Horizontal Laminae and Vertical Pore Canals in Rigid Cuticle of Tribolium castaneum. Insect Biochem. Mol. Biol. 2014, 53, 22-29.

(14) Park, D.; Wershof, E.; Boeing, S.; Labernadie, A.; Jenkins, R. P.; George, S.; Trepat, X.; Bates, P. A.; Sahai, E. Extracellular Matrix Anisotropy Is Determined by TFAP2C-Dependent Regulation of Cell Collisions. Nat. Mater. 2020, 19, 227-238.

(15) Kollmannsberger, P.; Bidan, C. M.; Dunlop, J. W. C.; Fratzl, P.; Vogel, V. Tensile Forces Drive a Reversible Fibroblast-toMyofibroblast Transition during Tissue Growth in Engineered Clefts. Sci. Adv. 2018, 4, No. eaao4881.

(16) Belamie, E.; Davidson, P.; Giraud-Guille, M. M. Structure and Chirality of the Nematic Phase in $\alpha$-Chitin Suspensions. J. Phys. Chem. B 2004, 108, 14991-15000.

(17) Bouligand, Y. Liquid Crystals and Biological Morphogenesis: Ancient and New Questions. C. R. Chim. 2008, 11, 281-296.

(18) Neville, A. C.; Luke, B. M. A Two-System Model for ChitinProtein Complexes in Insect Cuticles. Tissue Cell 1969, 1, 689-707.

(19) Neville, A. C. Circadian Organization of Chitin in Some Insect Skeletons. Q. J. Microsc. Sci. 1965, 106, 315-325.

(20) Moussian, B. Recent Advances in Understanding Mechanisms of Insect Cuticle Differentiation. Insect Biochem. Mol. Biol. 2010, 40, $363-375$.

(21) Çiçek, Ö.; Abdulkadir, A.; Lienkamp, S. S.; Brox, T.; Ronneberger, O. 3D U-Net: Learning Dense Volumetric Segmentation from Sparse Annotation. In Medical Image Computing and Computer-Assisted Intervention-MICCAI 2016; Ourselin, S.; Joskowicz, L.; Sabuncu, M. R.; Unal, G.; Wells, W., Eds.; Springer International Publishing: Cham, 2016; pp 424-432.

(22) Ronneberger, O.; Fischer, P.; Brox, T. U-Net: Convolutional Networks for Biomedical Image Segmentation. In Medical Image Computing and Computer-Assisted Intervention-MICCAI 2015; Navab, N.; Hornegger, J.; Wells, W. M.; Frangi, A. F., Eds.; Springer International Publishing: Cham, 2015; pp 234-241.

(23) Moussian, B.; Seifarth, C.; Müller, U.; Berger, J.; Schwarz, H. Cuticle Differentiation during Drosophila Embryogenesis. Arthropod Struct. Dev. 2006, 35, 137-152.

(24) Gorelik, J.; Shevchuk, A. I.; Frolenkov, G. I.; Diakonov, I. A.; Richardson, G. P.; Vodyanoy, I.; Edwards, C. R. W.; Klenerman, D.; Korchev, Y. E. Dynamic Assembly of Surface Structures in Living Cells. Proc. Natl. Acad. Sci. U.S.A. 2003, 100, 5819-5822.

(25) Blackwell, J.; Weih, M. A. Structure of Chitin-Protein Complexes: Ovipositor of the Ichneumon Fly Megarhyssa. J. Mol. Biol. 1980, 137, 49-60.

(26) Valverde Serrano, C.; Leemreize, H.; Bar-on, B.; Barth, F. G.; Fratzl, P.; Zolotoyabko, E.; Politi, Y.; Valverde, C.; Leemreize, H.; Bar-on, B.; et al. Ordering of Protein and Water Molecules at Their Interfaces with Chitin Nano-Crystals. J. Struct. Biol. 2016, 193, 124131.

(27) Tsuchiya, K.; Ishii, T.; Masunaga, H.; Numata, K. Spider Dragline Silk Composite Films Doped with Linear and Telechelic Polyalanine: Effect of Polyalanine on the Structure and Mechanical Properties. Sci. Rep. 2018, No. 3654.

(28) Sampath, S.; Isdebski, T.; Jenkins, J. E.; Ayon, J. V.; Henning, R. W.; Orgel, J. P. R. O.; Yarger, J. L.; Antipoa, O. X-ray Diffraction Study of Nanocrystalline and Amorphous Structure within Major and Minor Ampullate Dragline Spider Silks. Soft Matter 2012, 8, 67136722.

(29) Ling, S.; Li, C.; Adamcik, J.; Shao, Z.; Chen, X.; Mezzenga, R. Modulating Materials by Orthogonally Oriented $\beta$-Strands: Composites of Amyloid and Silk Fibroin Fibrils. Adv. Mater. 2014, 26, 45694574.

(30) Rudall, K. M. The Chitin/Protein Complexes of Insect Cuticles. Advances in Insect Physiology; Academic Press, 1963; Vol. 1, pp 257-313. 
(31) Murray, S. B.; Neville, A. C. The Role of PH, Temperature and Nucleation in the Formation of Cholesteric Liquid Crystal Spherulites from Chitin and Chitosan. Int. J. Biol. Macromol. 1998, 22, 137-144.

(32) Belamie, E.; Mosser, G.; Gobeaux, F.; Giraud-Guille, M. M. Possible Transient Liquid Crystal Phase during the Laying out of Connective Tissues: $\alpha$-Chitin and Collagen as Models. J. Phys.: Condens. Matter 2006, 18, S115.

(33) Narkevicius, A.; Steiner, L. M.; Parker, R. M.; Ogawa, Y.; Frkapetesic, B.; Vignolini, S. Controlling the Self-Assembly Behavior of Aqueous Chitin Nanocrystal Suspensions. Biomacromolecules 2019, 2830.

(34) Giraud-Guille, M.-M. Plywood Structures in Nature. Curr. Opin. Solid State Mater. Sci. 1998, 3, 221-227.

(35) Matsko, N.; Mueller, M. AFM of Biological Material Embedded in Epoxy Resin. J. Struct. Biol. 2004, 146, 334-343.

(36) Spong, K. E.; Mazzetti, T. R.; Robertson, R. M. Activity Dependence of Spreading Depression in the Locust CNS. J. Exp. Biol. 2016, 219, 626-630.

(37) Münch, B.; Trtik, P.; Marone, F.; Stampanoni, M. Stripe and Ring Artifact Removal with Combined Wavelet - Fourier Filtering. Opt. Express 2009, 17, 8567-8859.

(38) Limaye, A. In Drishti: A Volume Exploration and Presentation Tool, Proceedings of SPIE 8506, Developments in X-Ray Tomography VIII, 85060X, 2012.

(39) Benecke, G.; Wagermaier, W.; Schwartzkopf, C.; Li, M.; Flucke, G.; Hoerth, R.; Zizak, I.; Burghammer, M.; Metwalli, E.; MüllerBuschbaum, P.; Trebbin, M.; et al. DPDAK - A Customizable Software for Fast Reduction and Analysis of Large X-Ray Scattering Data Sets: Applications of the New DPDAK Package to Small Angle $\mathrm{X}$-Ray Scattering and Grazing-Incidence Small Angle X-Ray Scattering. J. Appl. Crystallogr. 2014, 47, 1797-1803.

(40) Anaconda, Computer Software; Anaconda Software Distribution, 2014. 